\title{
Respiratory Signal Prediction Based on Adaptive Boosting and Multi-Layer Perceptron Neural Network
}

\author{
W Z Sun ${ }^{1,2}, \mathbf{M}$ Y Jiang ${ }^{1}$, L Ren ${ }^{2}$, J Dang ${ }^{3}$, T You ${ }^{3}$, and F-F Yin ${ }^{2}$ \\ ${ }^{1}$ Institute of Information Science and Engineering, Shandong University, Shandong, People's \\ Republic of China \\ 2Department of Radiation Oncology, Duke University Cancer Center, Durham, NC, USA \\ ${ }^{3}$ Department of Radiation Oncology, Affiliated Hospital of Jiangsu University, Jiangsu, People's \\ Republic of China
}

\section{Abstract}

To improve the prediction accuracy of respiratory signals using adaptive boosting and multi-layer perceptron neural network (ADMLP-NN) for gated treatment of moving target in radiation therapy. The respiratory signals acquired using a Real-time Position Management (RPM) device from 138 previous 4DCT scans were retrospectively used in this study. The ADMLP-NN was composed of several artificial neural networks (ANNs) which were used as weaker predictors to compose a stronger predictor. The respiratory signal was initially smoothed using a SavitzkyGolay finite impulse response smoothing filter (S-G filter). Then, several similar multi-layer perceptron neural networks (MLP-NNs) were configured to estimate future respiratory signal position from its previous positions. Finally, an adaptive boosting (Adaboost) decision algorithm was used to set weights for each MLP-NN based on the sample prediction error of each MLP-NN. Two prediction methods, MLP-NN and ADMLP-NN (MLP-NN plus adaptive boosting), were evaluated by calculating correlation coefficient and root-mean-square-error between true and predicted signals. For predicting 500-ms ahead of prediction, average correlation coefficients were improved from 0.83 (MLP-NN method) to 0.89 (ADMLP-NN method). The average of rootmean-square-error (relative unit) for 500-ms ahead of prediction using ADMLP-NN were reduced by $27.9 \%$, compared to those using MLP-NN. The preliminary results demonstrate that the ADMLP-NN respiratory prediction method is more accurate than the MLP-NN method and can improve the respiration prediction accuracy.

\section{Keywords}

Radiation therapy; Dynamic tracking; Artificial neural network; Respiratory motion prediction; Multi-layer perceptron neural network

Disclosure of Conflicts of Interest

The authors have no relevant conflicts of interest to disclose. 


\section{Introduction}

Target motion, such as lung and liver tumors, during the process of radiation therapy may change substantially due to respiratory breathing pattern changes (Ernst F et al 2011, Torshabi A E et al 2013, Vergalasova I et al 2012). As a result, each radiation treatment field is typically designed to be larger than the actual target volume in order not to miss the target, which leads to unnecessary radiation exposure to normal tissues. A number of motion management methods have been extensively investigated: 1) Breath-hold method (Nelson C et al 2005, Murphy M J et al 2002, Nobuyoshi F and Yasutaka H 2014, Chen Z G 2015, Hunjan S et al 2008, Petralia G et al 2009) which requires patients holding their breath for a period of time during irradiation to minimize the respiratory motion. However, this technique may not be applicable for patients who cannot stably hold their breaths. 2) Free breathing treatments: patient breath freely during the treatment. Four-dimensional computed tomography (4D-CT) (Hong S et al 2011) is acquired during simulation to capture the tumor motion range to generate the internal target volume. The target volume is typically larger to account for the respiratory motion, and may lead to higher dose to the surrounding tissues. 3) Respiratory gated treatments: this method uses breathing signals received from an external system such as Real-time Position Management (RPM) to track the tumor motion and turn on the radiation beam when the target is within the beam's eye view (BEV) (Cai J et al 2013, Yan $\mathrm{H}$ et al 2006). 4) Dynamic tracking: the moving target is tracked dynamically by radiation beams so that the target is constantly within the BEV (Hansen R et al 2016). Both respiratory gating and dynamic tracking allow us to substantially reduce the treatment field to minimize the radiation damage to the normal tissue. Compared to respiratory gating, dynamic tracking has higher delivery efficiency since the radiation is continuously delivered while tracking the target.

Tracking the moving target requires understanding the target motion patterns/trajectories and predicting these motion trajectories ahead of treatment as the delivery system will need time to modify beams and field shapes in order to synchronize beam shape with target location. To predict the target position associated with the respiratory motion, a number of mathematical models were developed by other investigators. Among these methods, autoregressive integrated moving average (ARIMA) model (Babu C N et al 2014, Khashei M et al 2011), Kalman filtering method (Liu C Y et al 2014, Guan C et al 2013), and artificial neural network (ANN) (Seregni M et al 2013, Yan H et al 2006) were most commonly used. ARIMA model basically assumed that the relationship between prior- and future- respiratory signals was linear and fitted using a linear function. This method would not work well for respiratory signals with non-linear relationship which is apparently the nature of respiration. Kalman filtering method required establishing a state equation and an observation equation first. However, initial parameters in these equations were difficult to determine; and this method is more suitable for a linear function fitting between prior- and future- respiratory signals. On the other hand, the ANN method, especially multi-layer perceptron neuro-network (MLP-NN), provided effective prediction for both linear and nonlinear respiratory signals (Tsai $\mathrm{T}$ et al 2008). In fact, almost all breathing patterns are nonlinear as any breathing position at a time cannot be linearly represented from previous positions. However, the MLP-NN method tends to run into local minima and overfit, 
resulting in an inaccurate prediction. In this study, we developed a new algorithm called Adaboost MLP-NN (ADMLP-NN) to predict respiratory signals by incorporating Adaboost (Sun J et al 2011, Wu X D et al 2008) optimization method into the prediction algorithm. Although the Adaboost was used widely for other purposes, the novelty of this new algorithm is that it utilized several MLPs as weaker predictors and Adaboost as a decision algorithm to compose a strong predictor. The inclusion of Adaboost method reduces the risk of running into local minima and of overfits in the MLP-NN algorithm, and thus improves the precision and accuracy of prediction compared to the MLP-NN method. The effectiveness of these two methods (ADMLP-NN and MLP-NN) in the prediction of respiratory signals were tested utilizing 138 sets of clinic data obtained from a RPM system. The influence of different values used to set parameters in ADMLP-NN was also assessed in terms of their stability and reliability.

\section{Materials and methods}

\subsection{Data acquisition}

The respiratory breathing pattern was obtained by the RPM system in which an infrared tracking camera and reflective external markers were placed on the top of patient abdomen and were utilized to record the patient respiratory patterns with a sampling rate of $30 \mathrm{~Hz}$ (Varian Medical Systems, Palo Alto, CA) (Yan H et al 2006).

\subsection{Prediction process}

The general scheme for the prediction process based on respiratory breathing patterns using ADMLP-NN method is outlined in figure 1 and an example of the breathing signal timeseries diagram is as shown in figure 2. Each fully recorded breathing signal was preprocessed with normalization and divided into two components separated at time $\mathrm{K}$. The component prior to time $\mathrm{K}$, referred as the training signals, was first smoothed and then used for training to determine the weights $\left(w^{\prime} s\right)$ and biases $\left(b^{\prime} \mathrm{s}\right)$ in the MLP-NNs. The component after $\mathrm{K}$, referred as the testing signals, was used to evaluate the developed ADMLP-NN. The full breathing signals were normalized into the range $[-1,1]$ in order to reduce the training time and compare the results accurately. After being normalized, the signals were smoothed to reduce noises which had substantial effect on predicting results. In this study, a smoothing method called Savitzky-Golay (S-G) (Zawisza I et al 2014) algorithm was applied to denoise the respiratory signals. However, the testing signals weren't smoothed in order to keep the originality of raw data.

The AdaBoost method (as shown inside the dashed line box in figure 1), constructing a composite by sequentially training weak predictor (MLP-NN) while putting more and more emphasis on certain MLP-NNs, was used to predict respiratory signals. For each weak predictor, the training algorithm requires both training input (for prediction) and training output (for validation) data. The signal points between $\mathrm{t}_{\mathrm{t}}=1$ and $\mathrm{t}_{\mathrm{t}}=\mathrm{H}$ were used as training input data for predicting the target position at a specific time $\mathrm{t}_{\mathrm{t}}=\mathrm{H}+\mathrm{M}$ (training output). The training process would repeat and continue to predict the next target position. The signal points between $\mathrm{t}_{\mathrm{t}}=\mathrm{K}-\mathrm{H}-\mathrm{M}+1$ and $\mathrm{t}_{\mathrm{t}}=\mathrm{K}-\mathrm{M}$ were used in the training algorithm for predicting the final target position in the data set. In this study, the number of MLP-NNs was $T$. Each 
MLP-NN include weights and biases. Initial values for these weights and biases in each MLP-NN were set randomly. The cost function (as will be described in Equation 4) used in each MLP-NN was calculated and minimized by updated weights and biases through a backpropagation training method during the iteration process. This process would repeat until either the pre-set maximum iteration number or threshold value of the cost function was reached. Each pair of data points, i.e., input data $\mathrm{S}(1, \ldots, \mathrm{H})$ vs. output data $\mathrm{S}(\mathrm{H}+\mathrm{M})$, consists of a training data set. Then, the $q$ th probability distribution of each training data set for th $(t=1, \ldots, T)$ MLP-NN, $D_{t}(q)$, was introduced to indicate the concurrent probability of each training data set. The set with higher probability would occur more often than that with lower probability. Here $q$ was a value between 1 and $\mathrm{K}-\mathrm{M}-\mathrm{H}+1$ when the $\mathrm{K}-\mathrm{M}-\mathrm{H}$ training data set were used to train each MLP-NN. Whenever each MLP-NN training was completed, probability distribution $\mathrm{D}_{\mathrm{t}}(\mathrm{q})$ over the training data set would be updated according to the calculated absolute error between predict signals and test (true) signals of each MLP-NN (see Equation 6). Then, the th weaker predictor (MLP-NN) weight, $a_{t}$, was introduced to indicate the importance of th MLP-NN and was updated according to its probability distribution $\mathrm{D}_{\mathrm{t}}(\mathrm{q})$. This process would repeat until all $a$ ' $s$ were calculated.

Finally, the testing signals, denoted by $\mathrm{S}(\mathrm{K}+\mathrm{H}+\mathrm{M}+1, \mathrm{~K}+2, \ldots, \mathrm{K}+\mathrm{L})$, were utilized to validate the goodness of the trained ADMLP-NN. Similar to the process used in the training process, the signal points between $\mathrm{t}_{\mathrm{t}=\mathrm{K}+1}$ and $\mathrm{t}_{\mathrm{t}}=\mathrm{K}+\mathrm{H}$ were imported to the ADMLP-NN trained for predicting the target position, $\mathrm{S}^{\prime}(\mathrm{K}+\mathrm{H}+\mathrm{M})$, at a specific time $\mathrm{t}_{\mathrm{t}}=\mathrm{K}+\mathrm{H}+\mathrm{M}$. This process would also repeat and continue to predict the next target position. The signal points between $\mathrm{t}_{\mathrm{t}}=\mathrm{K}+\mathrm{L}-\mathrm{H}-\mathrm{M}+1$ and $\mathrm{t}_{\mathrm{t}}=\mathrm{K}+\mathrm{L}-\mathrm{M}$ were imported to the trained ADMLP-NN to predict the final target position $S^{\prime}(K+L)$. Finally, the predicting signals $S^{\prime}(K+H+M), \ldots, S^{\prime}(K+L)$ were compared with the true positions $\mathrm{S}(\mathrm{K}+\mathrm{H}+\mathrm{M}), \ldots, \mathrm{S}(\mathrm{H}+\mathrm{M})$. Besides, the signals between $\mathrm{K}$ and $\mathrm{K}+\mathrm{H}+\mathrm{M}$ were utilized to predict the first signal.

\subsection{Multi-layer perceptron neural network (MLP-NN)}

For a m-layer MLP-NN, the output of each training MLPt as shown in the dashed box of figure 1, was calculated as follows:

$$
y_{l}^{\varepsilon}(q)=f^{\varepsilon}\left(\sum_{j=1}^{n} w_{j l}^{\varepsilon} y_{j}^{\varepsilon-1}(q)+b_{l}^{\varepsilon}\right) \quad \varepsilon=2, \ldots, m
$$

Here, $w_{j l}^{\varepsilon}, b_{j}^{\varepsilon}, f^{\varepsilon}(x), j, l, q$ are the weights between current and fore-layer, biases, activation function, neuron number of fore-layer, neuron number of current layer and samples number of training input signals. Besides, $y_{l}^{\varepsilon-1}(q)$ is output of fore-layer and $y_{l}^{\varepsilon}(q)$ is the output of current layer. In this study, $m$ was 3 and neuro number of output layer was 1. Schematic diagram of the multi-layer perceptron neural network (MLP-NN) used in this study was outlined in figure 3 . The input, hidden and output layer were the first, second and third layer respectively. The functions shown in Equation 2 and Equation 3 were used as activation function of hidden layer and activation function of output layer, respectively. 


$$
f^{1}(x)=\frac{1}{1+e^{-x}}
$$

$$
f^{2}(x)=x
$$

After computing the output using random weights and biases, the cost function of MLP-NN using for evaluating the network training performance were calculated as:

$$
e(w, b)=\frac{1}{2} \sum_{q=H+M}^{K}\left(y_{1}^{3}(q)-S(q)\right)^{T}\left(y_{1}^{3}(q)-S(q)\right)
$$

Here, $S$ is true position and $y_{1}^{3}(q)$ is predicted position. An optimizing algorithm called Levenberg-Marquardt (LM) algorithm was applied to update weights and biases (Menhaj M B et all 2015).

\subsection{Adaboost algorithm}

The challenge of using MLP-NN algorithm is often associated with local minima and overfits. Therefore, an ADMLP-NN algorithm is developed to modify the MLP-NN algorithm. This is achieved by using a combination of several MLP-NNs with different weighting factors. For predicting the target position using Adaboost algorithm, the following steps were made:

1. After the first MLP-NN being developed, each training data set was assigned an initial probability distribution that obeys a uniform distribution.

$$
D_{1}(q)=\frac{1}{K-H-M+1} \quad q=H+M, H+M+1, \ldots, K
$$

Here, $D_{1}(q)$ was the first probability distribution for the th MLP-NN. $K-H-M+1$ was the number of training data set.

2. The normalized absolute error for each $q$ was calculated using follow formula:

$$
\begin{gathered}
\Theta_{t}(q)=\left|\frac{S_{t}^{\prime}(q)-S(q)}{E_{t}}\right| \\
E_{t}=\max \left|S_{t}^{\prime}(q)-S(q)\right|
\end{gathered}
$$

Here, $S$ were test signals, $S_{t}^{\prime}$ were predicted signals of th weaker predictor.

3. The total distribution of th MLP-NN $\xi_{t}$ was calculated using formula (8). 


$$
\xi_{t}=\sum_{q=H+M}^{K} D_{t}(q) * \Theta_{t}(q)
$$

4. The importance of each weaker predictor was represented by a weight $a_{t}$. The weight was calculated using follow formula.

$$
\alpha_{t}=\frac{\xi_{t}}{1-\xi_{t}}
$$

The probability distribution was updated according to the normalized absolute error between predict signals and test (true) signals of each MLP-NN which was regarded as the weaker predictor.

$$
D_{t+1}(q)=D_{t}(q) * \alpha_{t}^{1-\Theta_{t}(q)}
$$

Probability distribution of training data set of $t$ th MLP-NN was normalized:

$$
D_{t+1}(q)=\frac{D_{t+1}(q)}{\sum_{c=1}^{t+1} D_{c}(q)}
$$

5. Steps 2 to 4 were repeated until all parameters in all MLP-NNs were determined. Then, weaker predictor weights were normalized as follows:

$$
\alpha_{t}=\frac{\alpha_{t}}{\sum_{t=1}^{T} \alpha_{t}}
$$

6. The final predicted output of the ADMLP-NN was computed according to the sum of all weighted weaker predictors.

$$
S^{\prime}=\sum_{t=1}^{T} \alpha_{t} * S_{t}^{\prime}
$$

\subsection{Experiments and evaluation method}

A total of 138 sets of patients' data were used to test this method. Signals acquired around first 3 minutes were utilized as the training signals to train the neural network and the following 30s signals were used as test signals to assess the effectiveness of two prediction methods for both MLP-NN and ADMLP-NN. Unless otherwise specified, both ADMLP-NN and MLP-NN used the default settings: neuron number of input layer is 25 , neuron number of hidden layer is 8, initial learning rate is 0.001, number of MLP-NN used in ADMLP-NN is 8 , the threshold value for the cost function of MLP-NN is 0.05 and the time ahead of prediction is 500-ms (Lee S J et al 2012, Murphya M J et al 2009). 
In order to evaluate the performance of both ADMLP-NN and MLP-NN methods on different breathing patterns (King A P et al 2009), we defined a respiratory variability metric (irregular rate) used by (Huang L et al 2010, Cai J et al 2007) as the mean of the standard deviation (STD) in the maximal amplitude displacements (i.e., peaks) and in the minimal amplitude displacements (i.e., valleys). It can be calculated by Equation (14). All patient breathing patterns were then divided into two groups (regular and irregular) with equal number at the midterm variability value $v=0.22$.

$$
v=\frac{S T D(\text { peaks })+S T D(\text { valleys })}{2}
$$

root-mean-square-error (RMSE), correlation coefficient (CC) and maximum error (ME) between true and predicted signals were calculated as quantitative measures for assessment. For each test signal point, the absolute difference between the true and predicted values was calculated. RMSE was the mean of absolute differences from all predicted signal points. CC was used to test the degree of similarity between the true and prediction values over a period of time. The RMSE and CC were calculated as following.

$$
\begin{aligned}
& R M S E=\sqrt{\frac{1}{L-M-H+1} \sum_{q=H+M+K}^{K+L}\left(S^{\prime}(q)-S(q)\right)^{2}} \\
& C C=\frac{\sum_{q=H+M+K}^{K+L}\left(S^{\prime}(q)-\overline{\left.S^{\prime}(q)\right)(S(q)-\overline{S(q))}}\right.}{\sqrt{\sum_{q=H+M+K}^{K+L}\left(S^{\prime}(q)-{\overline{\left.S^{\prime}(q)\right)}}^{2}\right.} \sqrt{\sum_{q=H+M+K}^{K+L}(S(q)-\overline{S(q)})^{2}}} \\
& M E=\max \left|S^{\prime}(q)-S(q)\right|
\end{aligned}
$$

Here, $\overline{S(q)}$ is the average of true values, $\overline{S^{\prime}(q)}$ is the average of predicted values and $q$ ranges from $\mathrm{H}+\mathrm{M}+\mathrm{K}$ to $\mathrm{K}+\mathrm{L}$. Student's $\mathrm{t}$ test (T-test) was used as statistical method to assess the difference in this study. Statistical significance was considered with $\mathrm{p}<0.05$.

\section{Results}

Table 1 showed the average RMSEs, CCs and calculation times (CLTs) of 138 patients between the true and predicted signals using two prediction methods (ADMLP-NN and MLP-NN). Figures 4(a) and (b) illustrated RMSEs, MEs and CC calculated using two methods for each patient, respectively. Obviously, these values showed improvements of both RMSE and CC using ADMLP-NN. The CCs were increased from 0.86 (MLP-NN) to 0.91 (ADMLP-NN) for the regular group (average variability: $v=0.175$ ) and 0.80 (MLP-NN) to 0.87 (ADMLP-NN) for the irregular group (average variability: $v=0.292$ ), respectively. 
For the regular group, both RMSEs and MEs were reduced by $27 \%$ (from 0.22 to 0.16 ) and $16 \%$ (from 0.73 to 0.61 ), respectively. For the irregular group, both RMSEs and MEs were reduced by $32 \%$ (from 0.25 to 0.17 ) and $27 \%$ (from 0.89 to 0.65 ), respectively. The p-values of RMSE, ME and CC with 500-ms ahead of prediction for both regular and irregular groups were less than 0.001, indicating that the performance using ADMLP-NN was significantly improved compared to that using MLP-NN only. Besides, the training time of ADMLP-NN method was about 1.5-second which was about ten times longer than MLP-NN method using a personal computer (i7-6700HQ@2.6GHz 2.5GHz, 16G RAM).

Figure 5 illustrated sample all-time respiratory signals for one patient in the regular (a) and the irregular (c) groups as well as prediction performance using two methods in the regular (b) and the irregular (d) groups. The signals between the starting training point and $\mathrm{A}\left(\mathrm{t}_{\mathrm{t}}=1\right.$ and $t_{t}=K$ in Figure 2) were used to train the ANN model while the signals between B and C $\left(t_{t}=K+H+M\right.$ and $t_{t}=K+L$ in Figure 2) were used to evaluate the performance of two prediction methods. Besides, the signals between A and B were utilized to predict the first signal. Obviously, the ADMLP-NN method substantially improved the prediction performance compared to that using the MLP-NN method.

Impacts of some important parameters on the performance of the ADMLP-NN method were investigated and shown in table 2 in this study: 1) The neuron number of input layer $\mathrm{N}_{\mathrm{I}}$. The p-values of RMSE and CC for the first two pairs of $\mathrm{N}_{\mathrm{I}}$ (1 and 7, 7 and 13, 13 and 19) were less than 0.05 while the following two pairs (19 and 25) were more than 0.05. Both RMSE and $\mathrm{CC}$ were significantly improved as the $\mathrm{N}_{\mathrm{I}}$ increased to 19.2) The neuron number of hidden layer $\mathrm{N}_{\mathrm{H}}$. The effect of five different $\mathrm{N}_{\mathrm{H}}(1,2,4,6,8)$ on performance was investigated. As anticipated, proper selection of $\mathrm{N}_{\mathrm{H}}$ is important for the neural network performance. Too large $\mathrm{N}_{\mathrm{H}}$ may lead ANN to overfit. On the other hand, too small $\mathrm{N}_{\mathrm{H}}$ couldn't fit curve well. In this specific test, the number 8 provided the best performance. For both RMSE and CC, the p-values between each $\mathrm{N}_{\mathrm{H}}$ pair (1 and 2, 2 and 4, 4 and 6, 6 and 8) were less than 0.05 . The improvement was significant up to 8. 3) The initial learning rate $\mu$. The effect of $\mu$ on prediction result was investigated by selecting 5 different $\mu$ values $(0.001$, $0.01,0.1,1,10)$. The p-values of both RMSE and CC between each $\mu$ pair $(0.001$ and 0.01 , 0.01 and $0.1,0.1$ and 1, 1 and 10) were more than 0.05 , indicating that the performance of ADMLP-NN was moderately influenced by $\mu$ with no statistical significance. 4) The threshold value of the cost function (used in MLP-NN) G. 5 different values of G (0, 0.05, $0.1,0.15,0.2)$ were selected to evaluate the prediction performance. The iteration of MLP$\mathrm{NN}$ ended after its cost function $\varepsilon$ reach to $\mathrm{G}$ set in advance. In this experiment, the predicted results initially became better as the $G$ increased, but then became worse when $G$ > 0.05. The ADMLP-NN performed the best as the G equaled to 0.05. 5) The number of MLPNNs $\mathrm{N}_{\mathrm{M}}$. For the 138 patients, the p-value of RMSE and CC between 2 and 4, 4 and 6 were less than 0.05 . This indicated the difference was statistic significant when the $\mathrm{N}_{M}$ was less than or equal to 6 .

\section{Discussion}

An adaptive boosting technique of MLP-NN (ADMLP-NN) for predicting respiratory signals using prior signals was developed and tested. An evident improvement was found for 
predicting future motion with 500-ms. For both ADMLP-NN and MLP-NN methods, the averages of RMSE, ME and CC from the irregular group were worse than those from the regular group. It should be noted that prediction algorithms typically perform better for regular breathing patters compared to irregular breathing patterns. If active inspiration/ expiration makes breathing pattern regular, we believe the prediction results will improve. However, the performance improvement using the ADMLP-NN method was better in the irregular group than in the regular group. For the irregular respiratory signals, which are hard to be matched, the conventional MLP-NN method is prone to be trapped into local minima and overfit with the data, while the ADMLP-NN could substantially avoid these issues.

The influences of different parameters selected for the development of ADMLP-NN were also tested in this study. The prediction accuracy was improved initially with increasing the $\mathrm{N}_{\mathrm{I}}$. However, as the $\mathrm{N}_{\mathrm{I}}$ continued to increase, the prediction accuracy had minimal improvement and even became worse due to overfit for some patients. This could be related to the fact that, if the $\mathrm{N}_{\mathrm{I}}$ was too small, the breathing curve could not fit well. Therefore, increasing the $\mathrm{N}_{\mathrm{I}}$ by adding more features could initially improve the performance. However, if the $\mathrm{N}_{\mathrm{I}}$ was more than an optimal value, the performance of the network would degrade due to overfit and local minimum problems. The optimal number could be experimentally determined. In this study, it was found $\mathrm{N}_{\mathrm{I}}=25$ was the optimal choice for the patient data tested. We also noted that the effect of the $\mathrm{N}_{\mathrm{H}}$ was similar to the $\mathrm{N}_{\mathrm{I}}$. The performance improvement tended to decrease if the $\mathrm{N}_{\mathrm{H}}$ was too large, which led to overfit and to be trapped to local minimum values. In this study, $\mathrm{N}_{\mathrm{H}}=8$ was found to be an optimal value. There was weak correlation between the $\mu$ and the performance of ADMLP-NN according to table 2 . This could be related to the fact that, the $\mu$ is updated through the iterations (Hagan M T and Menhaj M B et al 1994). The $\mu$ will be increased by a constant factor when it results in a large error. Conversely, it will be reduced by a constant factor. The $\mathrm{G}$ value represents the accuracy requirement for the cost function $\mu$ of each MLP-NN as shown in Equation 4. If the neural network doesn't overfit, a smaller $\mathrm{G}$ usually indicated better performance. For the 138 patients' data in this study, the prediction performance initially became better with increased $G$ value at first, but then got worse when $G$ value > 0.05. Especially, when the G value was larger than 0.05, the accuracy of ADMLP-NN was dramatically decreased. Finally, the effect of the $\mathrm{N}_{\mathrm{M}}$ was tested in this study. For the 138 patients' data in this study, the $\mathrm{N}_{\mathrm{M}}$ had little effect on predicting performance when it was greater than or equal to 6 , which indicated that 6 layers of MLPs were adequate to predict the breathing curves for the patient data tested.

The limitation of this study was that the respiratory signals was based on RPM signal only, which could be different from the actual respiratory motion of internal organ. In future, we would try to evaluate the ADMLP-NN method on the real internal respiratory motion data. Besides, this method couldn't detect breathing pattern change. We are currently investigating on-line re-training method for predicting pattern changes. 


\section{Conclusion}

An ADMLP-NN method based on novel combination of multiple ANNs was developed to predict the future respiratory signals with 500-ms ahead, which could be used for dynamic tracking of moving target in radiation therapy.

\section{Acknowledgments}

This research was supported in part by the National Institutes of Health Grant No. R01 CA184173 and the Natural Science Foundation of Shandong Province, China (No. ZR2014FM039).

\section{References}

1. Ernst F, Schlaefer A, Schweikard A. Predicting the outcome of respiratory motion prediction. Medical physics. 2011; 38(10):5569-5581. [PubMed: 21992375]

2. Torshabi AE, Riboldi M, Fooladi AAI, Mosalla SMM, Baroni G. An adaptive fuzzy prediction model for real time tumor tracking in radiotherapy via external surrogates. Journal of Applied Clinical Medical Physics. 2013; 14(1):102-114.

3. Vergalasova I, Cai J, Yin F-F. A novel technique for markerless, self-sorted 4d-cbct: Feasibility study. Medical physics. 2012; 39(3):1442-1451. [PubMed: 22380377]

4. Nelson C, Starkschall G, Balter G, Fitzpatrick MJ, Antolak JA, Tolani N, Prado K. Respirationcorrelated treatment delivery using feedback-guided breath hold: A technical study. Medical physics. 2005; 32(1):175-181. [PubMed: 15719968]

5. Murphy MJ, Martin D, Whyte R, Hai J, Ozhasoglu C, Le QT. The effectiveness of breath-holding to stabilize lung and pancreas tumors during radiosurgery. International Journal of Radiation Oncology Biology Physics. 2002; 53(2):475-482.

6. Nobuyoshi F, Yasutaka H. Application of a deformable registration technique to investigate breathhold reproducibility. Medical physics. 2014; 32(12):700-707.

7. Chen ZG, Xu L, Zhang SW, Huang Y, Pan RH. Lesion discrimination with breath-hold hepatic diffusion-weighted imaging: A meta-analysis. World Journal of Gastroenterology. 2015; 21(5): 1621-1627. [PubMed: 25663782]

8. Hunjan S, Starkschall G, Rosen I, Prado K, Tolani N, Balter P. Comparison of breath-hold and freebreathing positions of an external fiducial by analysis of respiratory traces. Journal of Applied Clinical Medical Physics. 2008; 9(3):34-42.

9. Petralia G, Summers P, Viotti S, Montefrancesco R, Raimondi S, Bellomi M. Quantification of Variability in Breath-hold Perfusion CT of Hepatocellular Carcinoma: A Step toward Clinical Use. Medical physics. 2012; 265(2):448-456.

10. Hong S, Jung B, Ruan D. Real-time prediction of respiratory motion based on a local dynamic model in an augmented space. Physics in medicine and biology. 2011; 56(6):1775-1789. [PubMed: 21346273]

11. Cai J, Chang Z, O'Daniel J, Yoo S, Ge H, Kelsey C, Yin F-F. Investigation of sliced body volume (SBV) as respiratory surrogate. Journal of applied clinical medical physics. 2013; 14(1):3987. [PubMed: 23318383]

12. Yan H, Yin F-F, Zhu GP, Ajlouni M, Kim JH. The correlation evaluation of a tumor tracking system using multiple external markers. Medical physics. 2006; 33(11):4073-4084. [PubMed: 17153387]

13. Hansen R, Ravkilde T, Worm ES, Toftegaard J, Grau C, Macek K, Poulsen PR. Electromagnetic guided couch and multileaf collimator tracking on a TrueBeam accelerator. Medical physics. 2016; 43(5):2387-2398. [PubMed: 27147350]

14. Babu CN, Reddy BE. A moving-average filter based hybrid ARIMA-ANN model for forecasting time series data. Applied Soft Computing. 2014; 23:27-38.

15. Khashei M, Bijari M. A novel hybridization of artificial neural networks and ARIMA models for time series forecasting. Applied Soft Computing. 2011; 11(2):2664-2675. 
16. Liu CY, Shui PL, Wei G, Li S. Modified unscented Kalman filter using modified filter gain and variance scale factor for highly maneuvering target tracking. Journal of Systems Engineering and Electronics. 2014; 35(3):380-385.

17. Guan C, Luh PB, Michel LD, Chi ZY. Hybrid Kalman Filters for very short-term load forecasting and prediction interval estimation. IEEE Transactions on Power Systems. 2013; 28(4):3806-3817.

18. Seregni M, Pella A, Riboldi M, Orecchia R, Cerveri P, Baroni G. Real-time tumor tracking with an articial neural networks-based method: A feasibility study. Physica Medica. 2013; 29(1):48-59. [PubMed: 22209110]

19. Yan H, Yin F-F, Zhu GP, Ajlouni M, Kim JH. Adaptive prediction of internal target motion using external marker motion: a technical study. Physics in medicine and biology. 2006; 51(1):31-44. [PubMed: 16357429]

20. Tsai TI, Li DC. Approximate modeling for high order non-linear functions using small sample sets. Expert Systems with Applications. 2008; 34(1):564-569.

21. Sun J, Jia MY, Li H. AdaBoost ensemble for financial distress prediction: An empirical comparison with data from Chinese listed companies. Expert Systems with Applications. 2011; 38(8):93059312.

22. Wu XD, Kumar V, et al. Top 10 algorithms in data mining. knowledge and information systems. 2008; 14(1):1-37.

23. Zawisza I, Yan H, Yin F-F. Su-c-brf-07: A pattern fusion algorithm for multi-step ahead prediction of surrogate motion. Medical Physics. 2014; 41(6):98-98.

24. Wang T, Gao HJ, Qiu JB. A Combined Adaptive Neural Network and Nonlinear Model Predictive Control for Multirate Networked Industrial Process Control. IEEE Transactions on Neural Networks and Learning Systems. 2015; 27(2):416-425. [PubMed: 25898246]

25. Hagan MT, Menhaj MB. Training feedforward networks with the Marquardt algorithm. IEEE transactions on neural networks. 1994; 5(6):989-993. [PubMed: 18267874]

26. Huang L, Park K, Boike T, Lee P, Papiez L, Solberg T, Ding CX, Timmerman RD. A study on the dosimetric accuracy of treatment planning for stereotactic body radiation therapy of lung cancer using average and maximum intensity projection images. Radiotherapy and Oncology. 2010; 96(1):48-54. [PubMed: 20430460]

27. Cai J, Read PW, Baisden JM, Larner JM, Benedict SH, Sheng K. Estimation of error in maximal intensity projection-based internal target volume of lung tumors: a simulation and comparison study using dynamic magnetic resonance imaging. International Journal of Radiation Oncology Biology Physics. 2007; 69(3):895-902.

28. King AP, Rhode KS, Razavi RS, Schaeffter TR. An adaptive and predictive respiratory motion model for image-guided interventions: theory and first clinical application. IEEE Transactions on Medical Imaging. 2009; 28(18):2020-2032. [PubMed: 19666335]

29. Lee SJ, Motai Y, Murphy M. Respiratory motion estimation with hybrid implementation of extended Kalman Filter. IEEE Transactions on Industrial Electronics. 2012; 59(11):4421-4432.

30. Murphy MJ, Pokhrel D. Optimization of an adaptive neural network to predict breathing. Medical physics. 2009; 36(1):40-47. [PubMed: 19235372] 


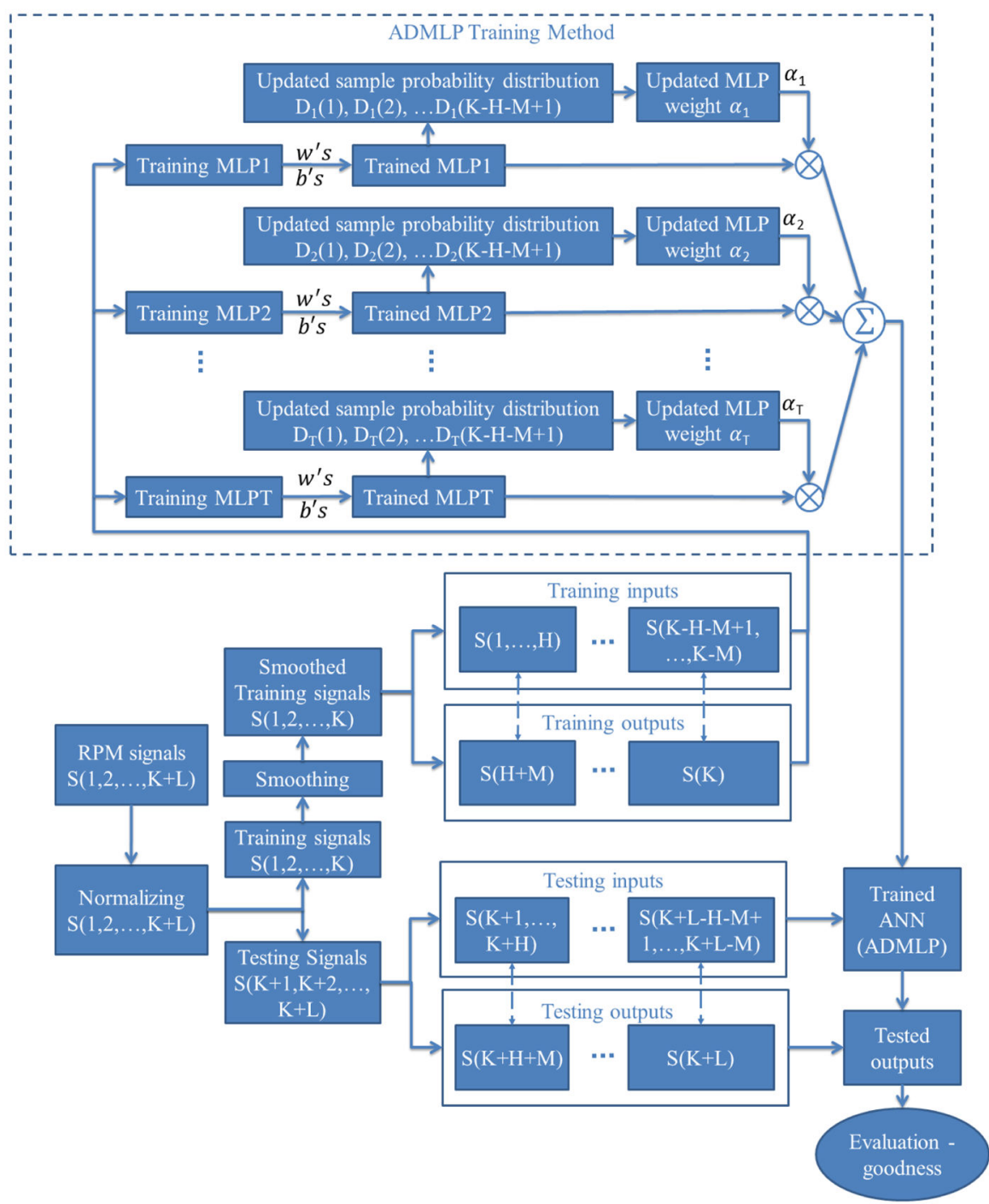

Figure 1.

Flow chart of the prediction algorithm. The dashed box indicating the ADMLP-NN training method. Symbol of $\times$ indicate the products of two elements and symbol of Sigma indicates the summation of all elements. 


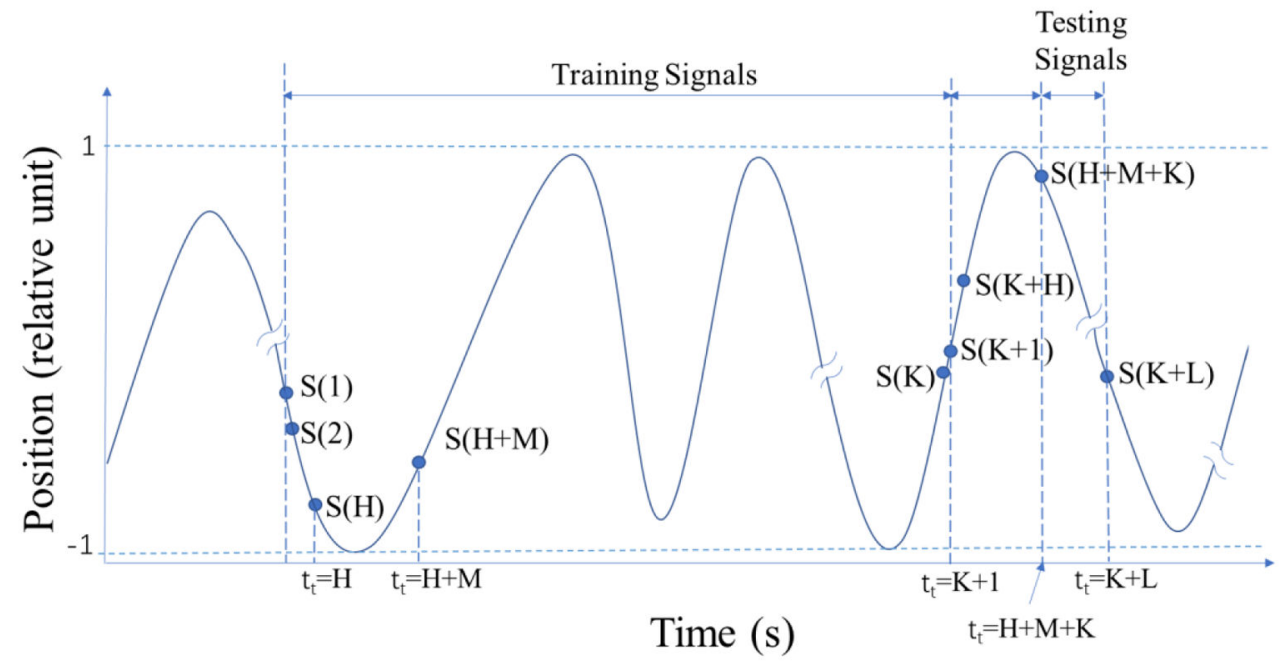

Figure 2.

Time-series diagram of respiratory breathing signals obtained from a Respiratory Positioning Management system. 


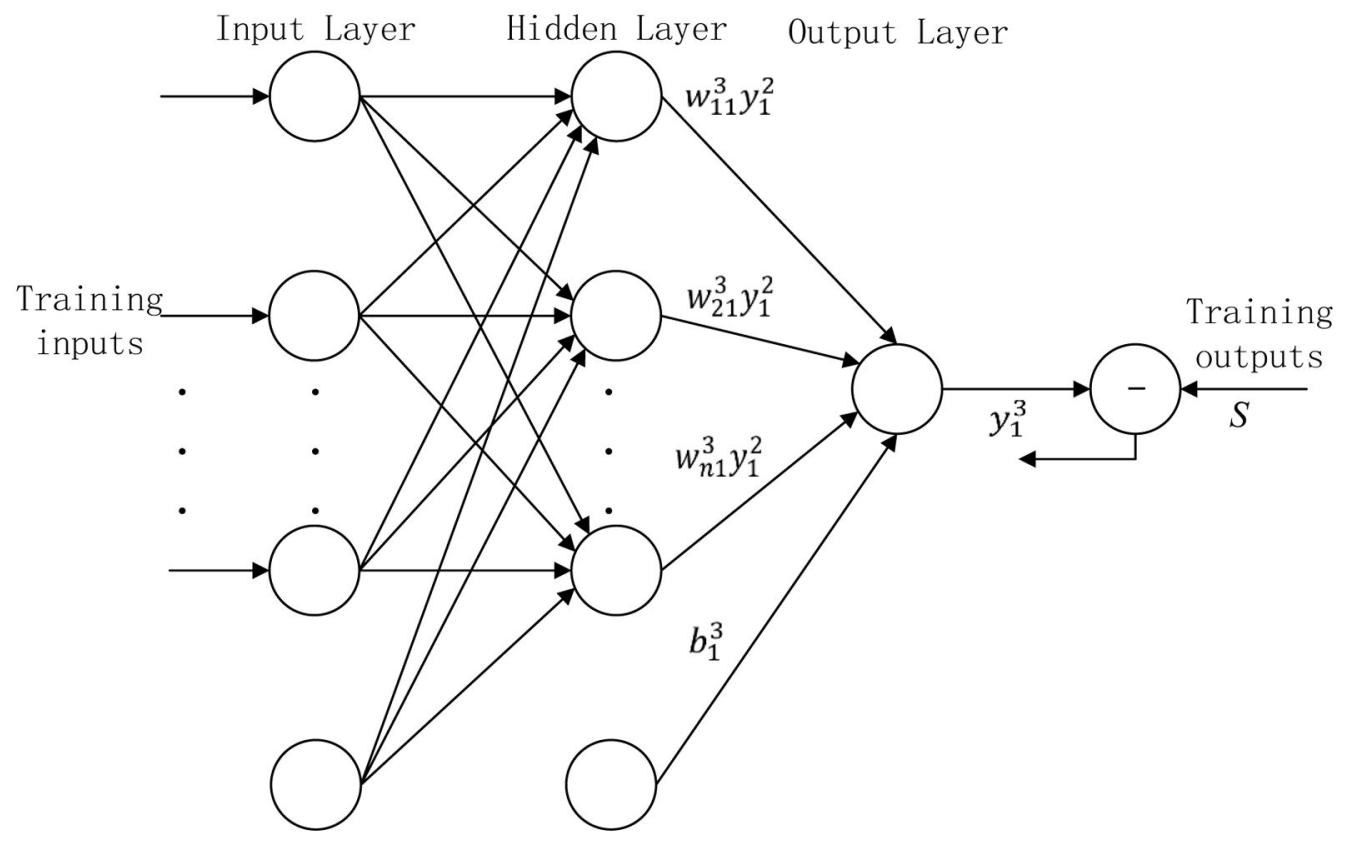

Figure 3.

Schematic diagram of the multi-layer perceptron neural network (MLP-NN) used for training purpose in this study 


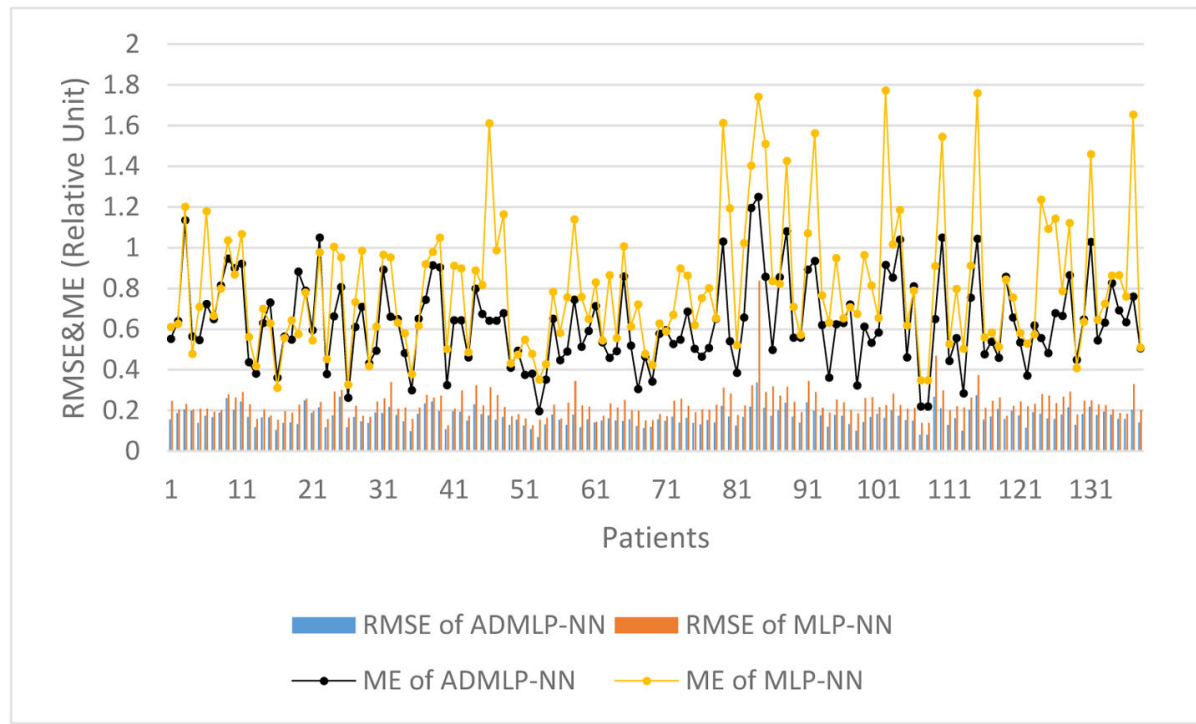

(a)

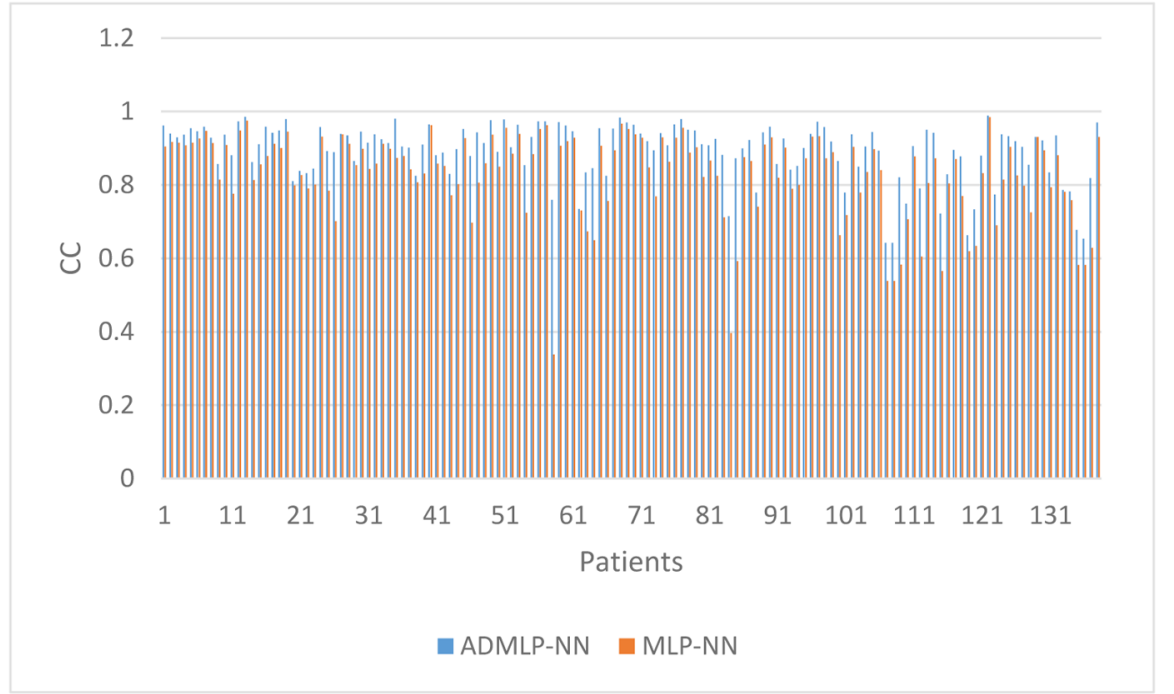

(b)

Figure 4.

Calculated RMSEs and MEs (a) as well as CCs (b) using both ADMLP-NN and MLP-NN methods for each patient 


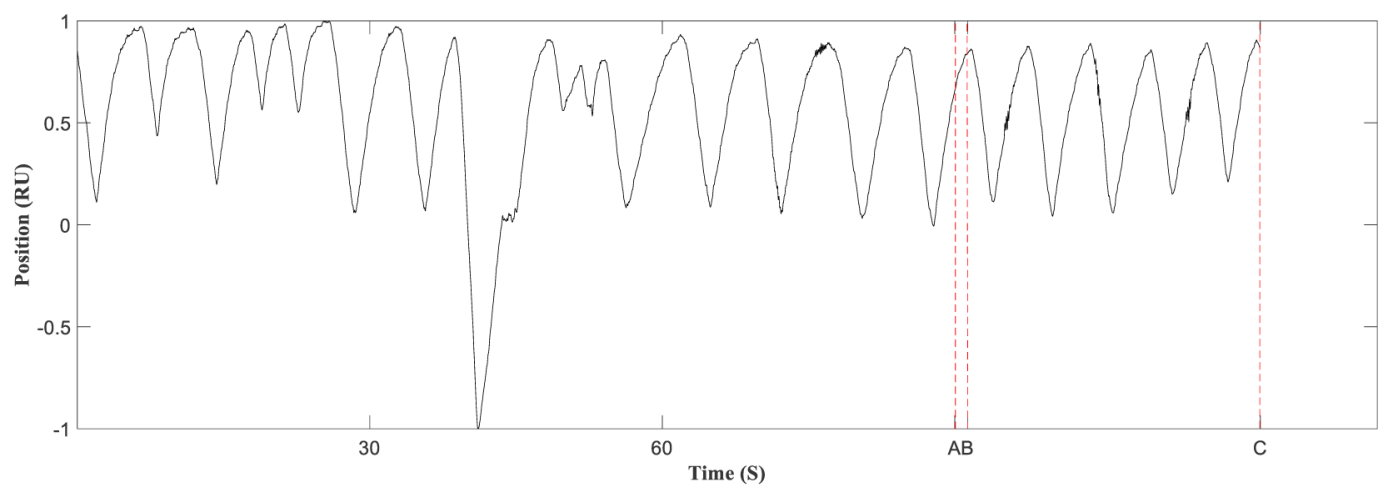

(a)

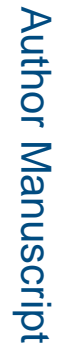

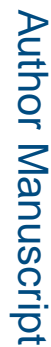

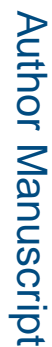




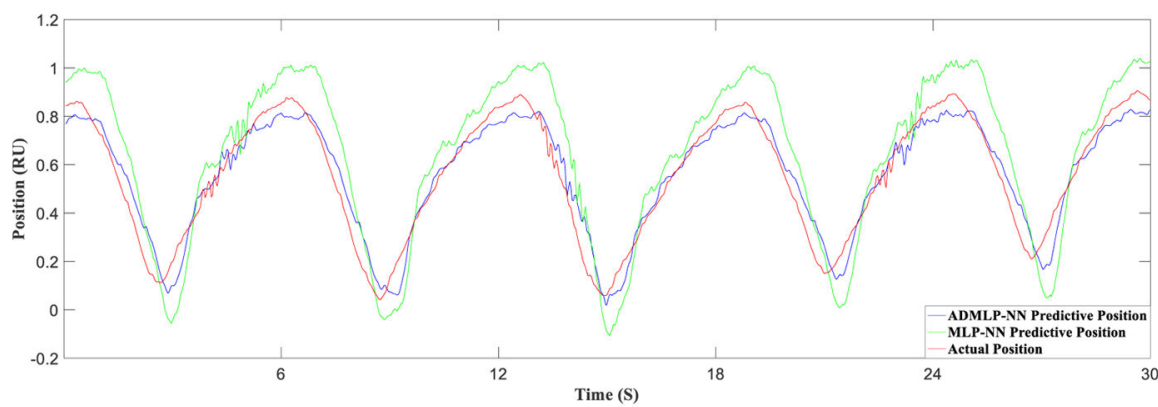

(b)

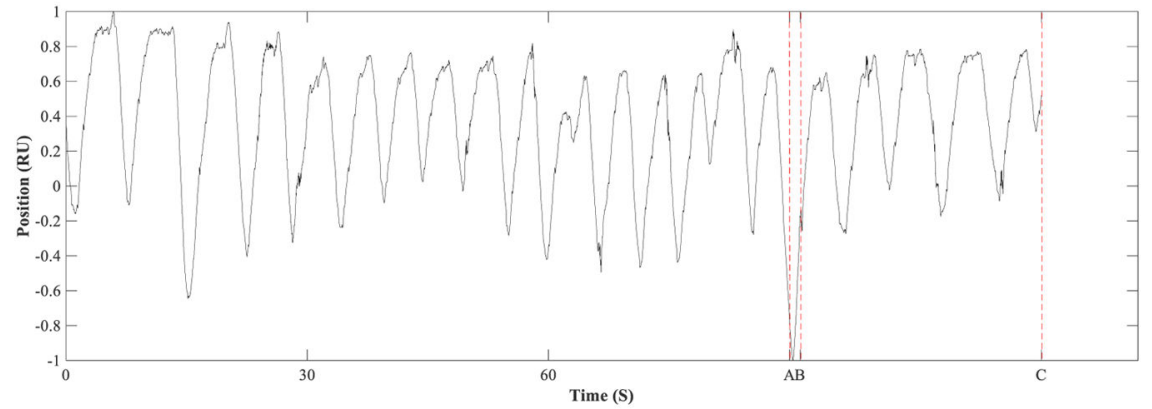

(c)

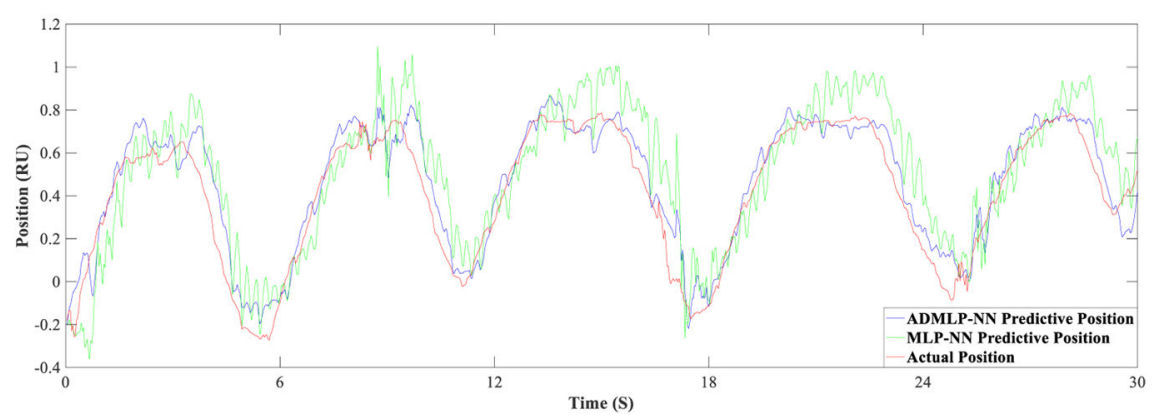

(d)

Figure 5.

Respiratory signals for one patient in (a) regular (c) irregular groups and prediction performance for two methods in (b) regular (d) irregular groups. 

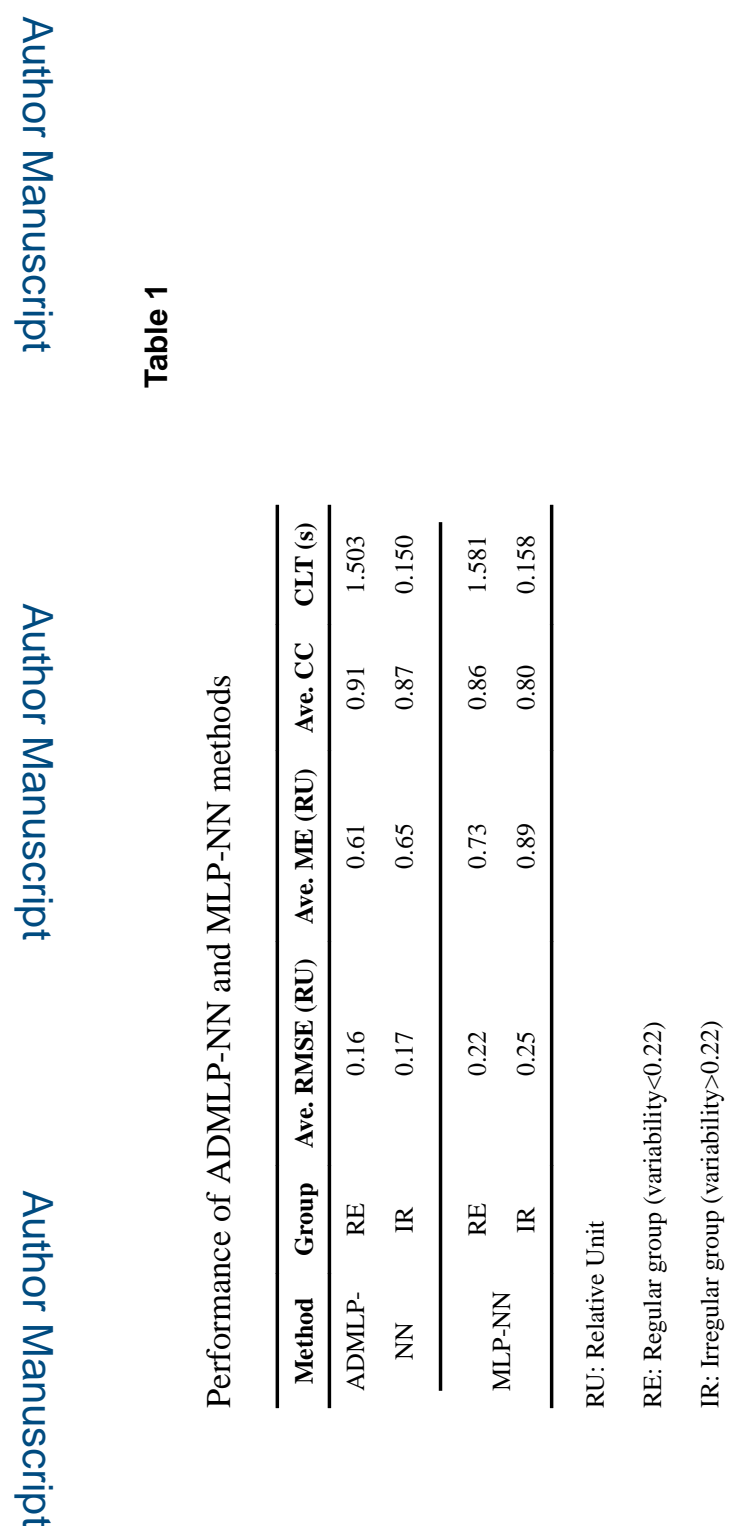
$\sum_{2}^{15}$

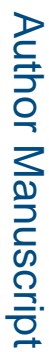

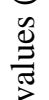

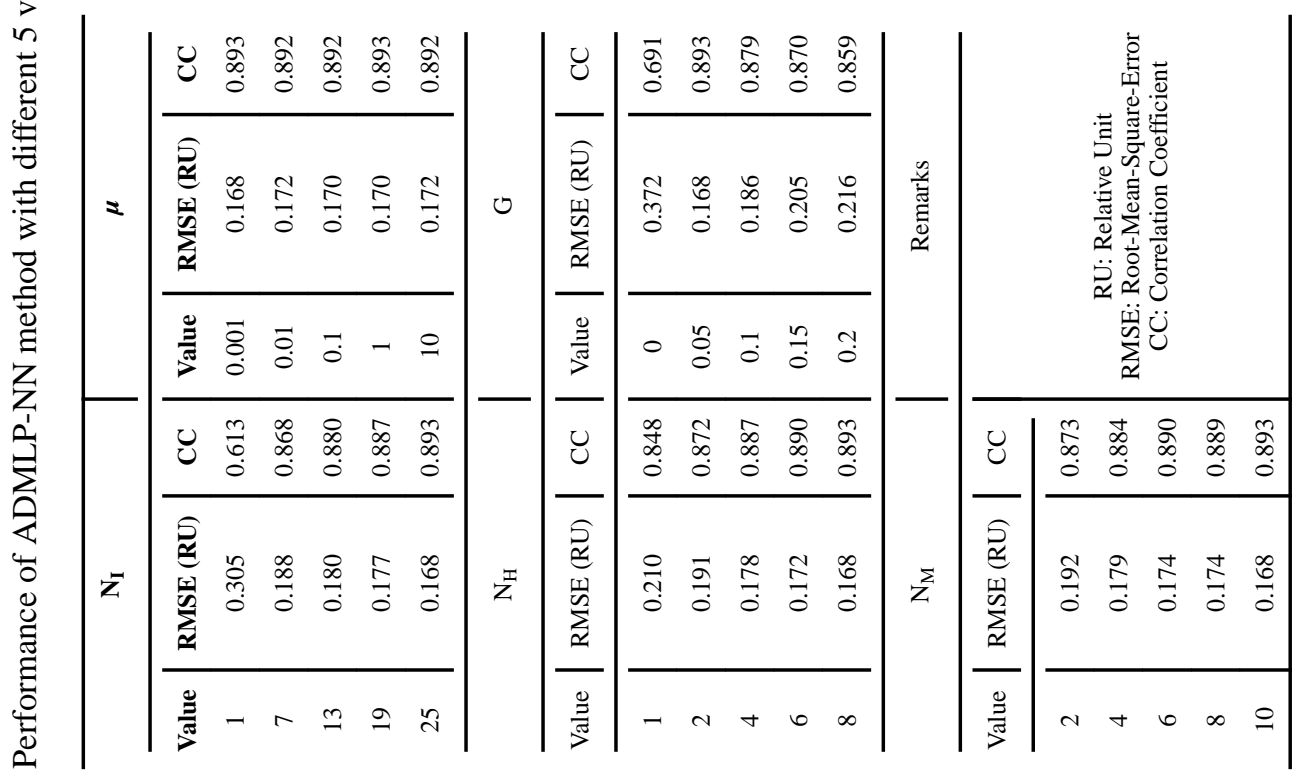

